\title{
QUALIDADE DE VIDA DA PESSOA DIABÉTICA
}

\author{
Tania Theodoro de Souza* \\ Luciana Santini* \\ Silvia Ayumi Wada* \\ Carolina Ferreira Vasco* \\ Miako Kimura**
}

SOUZA, T.T. de. et al. Qualidade de vida da pessoa diabética. Rev.Esc.Enf.USP, v.31, n.1, p. $150-64$, abr. 1997.

Trata-se de um estudo que teve por objetivos identificar o significado de qualidade de vida para a pessoa diabética, reconhecer os aspectos mais influenciados pela doença e o seu grau de satisfação com a vida. Participaram do estudo 46 pacientes diabéticos, adultos, de ambos os sexos, em tratamento ambulatorial. Os resultados obtidos demonstraram que o significado de qualidade de vida relacionouse, prioritariamente, ao bem estar físico $(54,5 \%)$, à estabilidade sócio-econômica (26,0\%) e ao bem-estar psico-emocional e espiritual (16,9\%). Os aspectos mais afetados pela doença foram: trabalho, estudo e atividades do lar $(38,5 \%)$, capacidade física $(25,6 \%)$ e relacionamento familiar $(10,3 \%)$. Quanto ao grau de satisfação com a vida, a maioria dos pacientes $(66,6 \%)$ considerou-se satisfeita ou muito satisfeita. Ressaltase a importância de que, na assistência à pessoa diabética, seja considerada a multidimensionalidade do conceito de qualidade de vida.

UNITERMOS: Qualidade de vida, Diabetes Mellitus.

\section{INTRODUÇÃO}

O diabetes mellitus é um problema universal, que vem afetando populações de vários países em todos os estágios de desenvolvimento. Nas últimas décadas, sua importância vem crescendo em decorrência de vários fatores, tais como: maior taxa de urbanização, aumento da esperança de vida, industrialização, sedentarismo, obesidade, dietas hipercalóricas e ricas em açúcares, entre outros (BRASIL, 1993).

\footnotetext{
* Alunas do $7^{\circ}$ semestre do curso de graduação em Enfermagem e Obstetrícia da Escola de Enfermagem da USP.

* Orientadora do trabalho. Professora Doutora do Departamento de Enfermagem MédicaCirúrgica da Escola de Enfermagem da USP.
} 
Segundo o Ministério da Saúde ( BRASIL,1993), devem existir na população brasileira cerca de 4.500 .000 diabéticos, dos quais aproximadamente 450.000 em uso de insulina. Além disso, estima-se que em torno de 2.000.000 de indivíduos desconhecem a sua condição de diabético, e que provavelmente, muitos só serão identificados ao apresentar uma condição crônica e irreversível da doença. Ressalta-se, ainda, um aumento da prevalência de diabéticos nas regiões Sul e Sudeste, que por serem as mais industrializadas, estão intimamente relacionadas às doenças crônico-degenerativas.

A média geral encontrada em nove capitais brasileiras $(7,60 \%)$ é pouco superior à observada na população dos Estados Unidos, que é de 6,6\%, onde o diabetes é considerado um problema de saúde pública. Vale a pena ressaltar que a magnitude da prevalência do diabetes desse país norte americano foi superada pelos resultados das cidades do Rio de Janeiro $(7,47 \%)$, São Paulo $(9,66 \%)$ e Porto Alegre (8,89\%), (BRASIL, 1993).

Estes dados tornam-se particularmente relevantes quando observamos que numa população economicamente instável como a brasileira, onde o sistema de saúde frequentemente convive com múltiplas crises financeiras e déficit na captação de recursos, existe um grande contingente de indivíduos com moléstias não diagnosticadas ou não tratadas adequadamente. Este fato tem levado a um aumento de internações hospitalares, assim como a uma elevada taxa de pagamentos de seguros e benefícios por incapacidade física.

A incapacidade física, no diabético, decorre do desenvolvimento de lesões crônicas nos vasos sanguíneos e nervos, afetando principalmente rins, retina, artérias, cérebro e nervos periféricos. Além disso, a pessoa diabética está sujeita a complicações de natureza aguda caracterizadas por crises de hipo ou hiperglicemia ( cetoacidose ), (CHA'TTON,1983; BEVILAQUA et al,1989; GREGG;ROBERTUS;STONE,1989; CHACRA,1991 ). Paralelamente, a pessoa diabética pode enfrentar dificuldades econômicas para adquirir alimentos e medicamentos, o que leva a problemas de adesão ao tratamento, bem como apresentar reações adversas à terapêutica adotada, contribuindo para o agravamento da doença. Exercem, também, papel importante no desenvolvimento de complicações a predisposição genética, doenças associadas como a hipertensão arterial e fatores ambientais, refletindo assim a somatória dos fatores psicossociais à própria fisiopatologia da doença.

$O$ diabetes mellitus é considerado uma doença crônica marcada por longa duração e recorrência frequente da sintomatologia. Embora as doenças crônicas tenham um caráter de permanência, nem todas são fatais ou desencadeadoras de maiores males, quando devidamente controladas (TRENTINI et al, 1990-a).

Adaptar-se de maneira bem sucedida à doença crônica inclui a concepção de que a quantidade e a qualidade da vida valem o esforço da luta (CURTIN; LUBKIN, 1990).

BURCKHARDT et al (1989); THOMPSON, (1990); HELLER, (1991); LIPP;ROCHA, (1994) definem como qualidade de vida o conjunto de respostas 
manifestadas pelos indivíduos aos fatores físicos, emocionais, sociais e econômicos com os quais interagem.GUTIÉRREZ (1985) explicita o conceito de qualidade de vida relacionando-o à capacidade de exercer funções da vida diária, à produtividade, à capacidade intelectual, à estabilidade emocional e à satisfação de viver. FERRANS; POWERS (1992) salientam que a satisfação com a vida, incluindo aspectos de interação familiar e social, desempenho físico e exercício profissional é um dos parâmetros importantes para a avaliação da qualidade de vida do indivíduo.

O paciente diabético é geralmente dependente de insulina ou hipoglicemiantes orais, de dieta especifica e exercícios físicos rigorosamente controlados (GREGG; ROBERTUS; STONE, 1989). Podemos, assim, pressupor que a repercussão da doença sobre o seu modo de vida seja bastante significativa, uma vez que a adaptação a ela requer todo um conjunto de mudanças nos hábitos e no cotidiano deste indivíduo ( CURTIN; LUBKIN, 1990).

$\mathrm{Na}$ tentativa de conhecer de forma mais global as condições de vida da pessoa diabética na convivência com sua doença, estabelecemos como objetivos especificos deste trabalho:

1. identificar o significado de "qualidade de vida" para a pessoa diabética;

2. reconhecer em que aspectos de sua vida o diabetes causou maior influência;

3. identificar o seu grau de satisfação com a vida .

\section{METODOLOGIA}

\subsection{Local de Pesquisa}

O presente estudo foi realizado no Ambulatório de Clínica Médica de dois hospitais gerais, páblicos e de ensino do Município de São Paulo.

\subsection{População de estudo}

Foram incluídos no estudo 46 pacientes diabéticos, adultos, de ambos os sexos, vinculados a grupos especificos de tratamento do diabetes mellitus, grupos estes integrados aos ambulatórios dos hospitais citados.

Os critérios de inclusão dos pacientes foram: estar presentes no ambulatório para consulta médica nos dias determinados para coleta de dados e concordar em participar do estudo.

\subsection{Coleta de Dados}

\subsubsection{Instrumento}

As informações pertinentes ao estudo foram obtidas por meio de um formulário composto por três partes (ANEXO I). A primeira parte incluiu os 
seguintes dados de identificação: nome, idade, sexo, estado civil, escolaridade, ocupação/profissão, situação empregatícia, renda familiar e número de dependentes. $\mathrm{Na}$ segunda, constaram dados relativos ao diagnóstico e tratamento, como: tipo de diabetes, sinais, sintomas e complicações e regime de tratamento atual. A terceira parte destinou-se à obtenção de dados especificos sobre qualidade de vida e o grau de satisfação com a mesma, na perspectiva dos pacientes entrevistados. $O$ instrumento foi submetido a um teste prévio, com ajustes necessários quanto ao seu conteúdo.

\subsubsection{Procedimento}

A coleta de dados foi realizada em outubro de 1994, após autorização formal das chefias de enfermagem dos ambulatórios, campos de pesquisa.

Os formulários foram preenchidos mediante entrevista realizada pelas autoras do estudo, no próprio ambulatório, durante o periodo que antecedeu a consulta médica ou após a mesma. Ao primeiro contato, as entrevistadoras identificavam-se junto ao paciente, esclarecendo-o sobre os objetivos do estudo e o caráter facultativo de sua participação. Os registros dos depoimentos foram feitos durante as entrevistas, cujo tempo médio de duração foi de 20 minutos.

\subsection{Tratamento Estatístico}

Os dados foram analisados em números absolutos e percentuais.

\section{RESULTADOS}

\subsection{Dados de Identificação}

Tabela 1. Distribuição dos pacientes entrevistados segundo idade e sexo. São Paulo, 1994

\section{SEXO}

\begin{tabular}{cccccrr} 
Idade & \multicolumn{2}{c}{ Masculino } & \multicolumn{2}{c}{ Feminino } & \multicolumn{2}{c}{ TOTAL } \\
& $\mathrm{N}^{\mathrm{o}}$ & $\%$ & $\mathrm{~N}^{\circ}$ & $\%$ & \multicolumn{1}{c}{$\mathrm{N}^{\circ}$} & \multicolumn{1}{c}{$\%$} \\
\hline $20-30$ & 3 & 6,5 & 2 & 4,3 & 5 & 10,9 \\
$31-40$ & 2 & 4,3 & 2 & 4,3 & 4 & 8,7 \\
$41-50$ & 7 & 15,2 & 1 & 2,2 & 8 & 17,4 \\
$51-60$ & 8 & 17,4 & 7 & 15,2 & 15 & 32,6 \\
$61-70$ & 6 & 13,0 & 6 & 13,0 & 12 & 26,1 \\
$71-80$ & 1 & 2,2 & 1 & 2,2 & 2 & 4,3 \\
\hline TOTAL & $\mathbf{2 7}$ & $\mathbf{5 8 , 7}$ & $\mathbf{1 9}$ & $\mathbf{4 1 , 3}$ & $\mathbf{4 6}$ & $\mathbf{1 0 0 , 0}$ \\
\hline
\end{tabular}


Pelos dados da Tabela 1, observa-se que, dos 46 pacientes entrevistados, 27 (58,7\%) eram do sexo masculino e $19(41,3 \%)$ do sexo feminino. Para ambos os sexos, a faixa etária predominante foi a compreendida entre 51 a 70 anos $(58,7 \%)$, sendo que $32,6 \%$ dos pacientes tinham idade entre 51 e 60 anos. A maior frequência de pacientes com idade superior a 50 anos corrobora os dados estatísticos do Ministério da Saúde (BRASIL, 1983), que constataram um aumento da frequência do diabetes mellitus após esta idade, em razão da crescente tendência de envelhecimento populacional observado atualmente no país.

Em relação ao estado civil, a grande maioria dos pacientes era casada $(67,4 \%)$, havendo também, em igual frequência, pacientes solteiros e viúos $(13,0 \%)$. Os demais $(6,6 \%)$ eram separados.

No que se refere ao número de dependentes, $41,3 \%$ dos entrevistados tinham entre 2 e 3 dependentes, $37,0 \%$ possuiam 1 ou nenhum, $17,4 \%$, de 4 a 5 e $4,3 \%$, mais de 05 dependentes.

Quanto a escolaridade, o maior contingente $(60,9 \%)$ era de pacientes não alfabetizados ou com primeiro grau incompleto; $8,7 \%$ dos pacientes informaram ter concluído o primeiro grau, havendo também pacientes que cursaram até o segundo e terceiro graus $(17,4 \%$ e $13,0 \%$ respectivamente).

Para a análise das ocupações utilizou-se a classificação proposta por FONSECA (1967), com adaptações para este trabalho.

Com este referencial, observamos a predominância de pacientes com ocupações de natureza manual, especializadas ou não $(65,2 \%) ; 26,1 \%$ dos pacientes não tinham ocupação definida, sendo incluídos nesta categoria, os estudantes, desempregados e aponsentados. Um dos pacientes $(2,2 \%)$ não informou sua ocupação.

Com relação à renda familiar, 05 pacientes não souberam informar sobre este dado. Com base nas informações de 41 pacientes, verificou-se um predomínio daqueles com renda compreendida entre 01 e 03 salários mínimos (SM) (34,0\%); $27,0 \%$ dos informantes recebiam entre 07 e $09 \mathrm{SM}$, seguidos de $22,0 \%$ que tinham rendimento de 10 ou mais $\mathrm{SM}$, e de $17,0 \%$ com uma renda de 04 a $06 \mathrm{SM}$.

Segundo o Ministério da Saúde ( BRASIL, 1993), o diabetes mellitus acomete igualmente todos os níveis sócio-econômicos da população, não sendo uma doença das classes mais favorecidas como habitualmente se pensa.

\subsection{Dados do diagnóstico e tratamento}

Para análise dos tipos de diabetes, os pacientes foram categorizados em dois grandes grupos: os diabéticos do tipo I, ou insulino-dependentes e os do tipo II, não insulino-dependentes (CHACRA, 1991). Na população entrevistada predominaram os do tipo II, equivalendo a $78,3 \%$ dos pacientes, enquanto que os do tipo I corresponderam a $21,7 \%$. Estatísticas revelam que, entre os diabéticos em geral, o diabetes do tipo II é sempre mais frequente do que o do tipo I, fato 
que também ocorreu entre a população do presente estudo. Segundo BRUNNER; SUDDARTH (1985), o diabetes mellitus insulino-dependente (DMID) corresponde a um percentual entre $5 \%$ a $10 \%$ de todos os diabéticos e o não insulinodependente (DMNID), entre $90 \%$ a $95 \%$.

Em relação ao tempo de diagnóstico, prevaleceram 29 pacientes com até 10 anos de identificação da doença $(66,0 \%)$ e 10 , com 20 anos de diagnóstico (23,0\%). Houve 02 pacientes $(4,5 \%)$ com diagn6stico estabelecido há menos de 01 ano; $02(4,5 \%)$ há 40 anos e 01 paciente $(2,0 \%)$ diagnosticado há 50 anos, dados estes que confirmam a cronicidade da doença.

Tabela 2. Sinais e sintomas relatados pela população de estudo. São Paulo, 1994

\begin{tabular}{|c|c|c|}
\hline Sinais e Sintomas & $\mathbf{N}^{\mathbf{o}}$ & $\%$ \\
\hline Polidipsia & 27 & 28,0 \\
\hline Poliúria & 19 & 19,6 \\
\hline Cansaço/Fraqueza & 11 & 11,3 \\
\hline Emagrecimento & 08 & 8,2 \\
\hline Náuseas/Mal estar & 07 & 7,2 \\
\hline Sudorese & 07 & 7,2 \\
\hline Desmaio & 07 & 7,2 \\
\hline Diminuição/Perda função visual & 06 & 6,2 \\
\hline Polifagia & 05 & 5,1 \\
\hline TOTAL & 97* & 100,0 \\
\hline
\end{tabular}

* Média de 2.1 indicações por paciente.

Observa-se, na Tabela 2, que os sinais e sintomas mais freqüentemente relatados pela população de estudo foram a polidipsia $(28,0 \%)$ e a poliúria $(19,6 \%)$, seguidas de cansaço/fraqueza ( $11,3 \%)$ e emagrecimento $(8,2 \%)$. Tais problemas, assim como os demais mencionados na Tabela 2 , são típicas manifestações do diabetes mellitus, podendo ocorrer de forma isolada ou simultânea (MARCONDES, 1989).

Indagados sobre o regime do tratamento atual, 23 pacientes $(50,0 \%$ dos entrevistados) referiram a utilização de insulina; $18(39,1 \%)$, a utilização de hipoglicemiantes orais; 03 pacientes $(6,5 \%)$ utilizavam simultaneamente insulina e hipoglicemiantes orais e apenas 02 pacientes $(4,4 \%)$ não necessitavam de terapêutica medicamentosa, controlando o diabetes somente com dieta e/ou exercício físico (caminhada). 


\subsection{Dados sobre Qualidade de Vida e Grau de Satisfação}

Tabela 3. Categorias de significados de qualidade de vida atribuídos pela população de estudo. São Paulo, 1994

\begin{tabular}{lcr}
\hline \multicolumn{1}{c}{ Categorias de significados } & $\mathbf{N}^{\mathbf{0}}$ & \% \\
\hline Bem-estar físico & 42 & 54,5 \\
Estabilidade sócio-econômica & 20 & 26,0 \\
Bem-estar psico-emocional e espiritual & 13 & 16,9 \\
Outros (lazer e auto-estima) & 02 & 2,6 \\
\hline \multicolumn{1}{c}{ TOTAL } & & $\mathbf{1 0 0 , 0}$ \\
\hline
\end{tabular}

* Média de 1,7 indicações por paciente.

Os dados da Tabela 3 demonstram que, para as pessoas diabéticas deste estudo, o significado de qualidade de vida foi relacionado, principalmente, ao seu bem-estar físico $(54,5 \%$ das indicações). Foram considerados como indicativos desta categoria, "viver com saúde" $(31,1 \%)$, "ter condições de trabalhar" $(10,3 \%)$ , "conseguir controlar a doença" $(7,9 \%)$ e "poder alimentar-se sem restrições" $(5,2 \%)$. A menção de tais aspectos parece indicar que, para os pacientes deste estudo, o bem-estar físico pode ser interpretado como ausência da doença ou de seus sintomas e limitações.

Segundo TRENTINI et al (1990-b), a qualidade de vida pode ser influenciada pela percepção que um indivíduo tem sobre seu estado de saúde. Esta afirmação reforça o fato de que, para os entrevistados, "estar saudável" foi um fator considerado importante para sua qualidade de vida.

O segundo aspecto mais citado foi o sócio-econômico com $26,0 \%$ das indicações. Dentre estas, ter estabilidade financeira (19,5\%) prevaleceu como a mais frequente. A possibilidade de estar inserido em um trabalho remunerado é um fator fundamental, não somente para a segurança e a auto-estima dos indivíduos, mas também para garantir a sua sobrevivência e a de seus dependentes. $O$ outro significado atribuído à qualidade de vida pelos pacientes foi o bem-estar psico-emocional e espiritual $(16,9 \%)$, vinculado ao fato de terem relacionamento familiar harmônico (10,4\%), paz espiritual $(3,9 \%)$ e apoio religioso $(2,6 \%)$. Lazer e auto-estima foram também mencionados como aspectos relacionados à qualidade de vida. Concordamos com CURTIN; LUBKIN (1990) na afirmação de que todos estes aspectos (físico, emocional, espiritual e sócioeconômico) não podem ser dissociados quando se pensa em qualidade de vida, pois são partes inter-relacionadas e interdependentes da condição humana. 
Tabela 4. População de estudo, segundo aspectos mais influenciados pelo diabetes. São Paulo, 1994

\begin{tabular}{llc}
\hline \multicolumn{1}{c}{ Aspectos } & $\mathbf{N}^{\mathbf{0}}$ & $\%$ \\
\hline Trabalho/estudo/atividades do lar & 15 & 38,5 \\
Capacidade física & 10 & 25,6 \\
Relacionamento familiar & 04 & 10,3 \\
Alimentação & 03 & 7,7 \\
Independência/auto-cuidado & 02 & 5,1 \\
Recreação e lazer & 02 & 5,1 \\
Outros (segurança no atendimento médico, & 03 & 7,7 \\
sexualidade e auto-imagem) & & \\
\hline \multicolumn{1}{c}{ TOTAL } & $\mathbf{3 9}$ & $\mathbf{1 0 0 , 0}$ \\
\hline
\end{tabular}

Dos 46 pacientes entrevistados, 07 ( $15,2 \%)$ informaram que não houve influência do diabetes em quaisquer dos aspectos de sua vida. Assim, os dados desta Tabela referem-se aos 39 pacientes que, de alguma forma, sofreram alterações em decorrência da doença. Podemos observar que a área relacionada ao trabalho, estudo e atividades do lar foi referida por 15 pacientes $(38,5 \%)$, para os quais a doença influenciou causando limitação física, necessidade de ausentar-se ou retirar-se mais cedo do trabalho, problemas visuais, nervosismo e conflitos com o patrão, havendo ainda aqueles que referiram problemas sócioeconômicos devido ao afastamento do trabalho.

A capacidade física foi a segunda área mais influenciada pelo diabetes, citada por 10 pacientes $(25,6 \%)$. As justificativas apontadas para esta influência foram a diminuição da capacidade e o cansaço físico, além de emagrecimento, fraqueza e desânimo.

Embora em menor freqüência, outros aspectos também foram citados, como relacionamento familiar (10,3\%); alimentação, (7,7\%); recreação/lazer (5,1\%) e independência/auto-cuidado $(5,1 \%)$. A assistência médica, sexualidade e autoimagem também foram citados por 3 entrevistados $(7,7 \%)$.

De acordo com estudo de TRENTINI et al $(1990$ - b), os indivíduos com doenças crônicas (entre elas o diabetes) e suas famílias, deverão adaptar-se a várias mudanças tais como perda na capacidade física e no trabalho.

A partir dos resultados observados na Tabela 4 , pressupomos que a confirmação de uma condição crônica de doença tende a afetar aspectos sociais, psicológicos, físicos e econômicos da vida de uma pessoa. Problemas como conflito de independência e auto cuidado, auto-imagem, alimentação e alteração na sexualidade também são fatores inevitavelmente enfrentados por pacientes portadores do diabetes. O objetivo da terapêutica para a maioria dos indivíduos em condições crônicas não é a cura, maśs sim a redução da severidade da doença ou a detenção de sua progressão. Estes indivíduos só poderão alcançar essa 
meta se enfrentarem da maneira mais positiva possível as inúmeras condições inerentes à situação de pessoa com doença crônica, as quais incluem: conhecer a doença, modificar sua rotina diária, ajustar-se aos relacionamentos sociais, enfrentar perdas, adaptar-se a mudanças no papel social, seguir o tratamento e controlar os sintomas físicos.

Acreditamos que fatores como o tempo de contato com o paciente e o próprio ambiente da coleta de dados possam, de alguma forma, ter influenciado nas respostas dos entrevistados. Entretanto, foi interessante observar uma indicação espontânea da sexualidade como área afetada pelo diabetes. Sabe-se que a cronicidade da doença pode ter sério impacto na esfera sexual, o que pode resultar em disfunção. Ocasionalmente, a impotência sexual pode ser um dos primeiros sintomas que leva à suspeita de que o paciente seja diabético. A incidência de impotência entre as pacientes com diabetes de longo período é de 50 a $60 \%$ (KOLODNY et al., 1974; CAMPBELL; CLARK, 1975 apud GREGG; ROBERTUS;STONE, 1989).

Tabela 5. Distribuição dos pacientes segundo o seu grau de satisfação com a vida. São Paulo, 1994

\begin{tabular}{|c|c|c|}
\hline Grau de satisfação & $\mathbf{N}^{\mathbf{o}}$ & $\%$ \\
\hline Satisfeito & 18 & 40,0 \\
\hline Muito satisfeito & 12 & 26,6 \\
\hline Indiferente & 7 & 15,6 \\
\hline Insatisfeito & 7 & 15,6 \\
\hline Muito insatisfeito & 1 & 2,2 \\
\hline TOTAL & 45 & 100,0 \\
\hline
\end{tabular}

Dentre os 46 pacientes, um não respondeu sobre o seu grau de satisfação com a vida. Dos 45 respondentes, $30(66,6 \%)$ consideraram-se muito satisfeitos ou satisfeitos. Houve 07 indivíduos $(15,6 \%)$ que se disseram indiferentes, e outros $07(15,6 \%)$,insatisfeitos; apenas 01 entrevistado $(2,2 \%)$ considerou-se muito insatisfeito.

Pesquisas prévias têm verificado que o grau de satisfação com a vida está relacionado com a quantidade e qualidade de interações sociais, satisfação com os relacionamentos conjugais, condições sócio-econômicas, estado de saúde, grau de independência, oportunidade de selecionar metas pessoais, e disponibilidade de recursos para alcançar os objetivos pessoais (GREGG; ROBERTUS; STONE, 1989). Isto pôde ser constatado no presente estudo, pois aqueles que consideraram-se muito satisfeitos ou satisfeitos $(66,6 \%)$ apresentaram justificativas relacionadas ao fato de ter saúde e o diabetes controlado; ter relação familiar harmônica; conseguir suprir as necessidades da família, estar trabalhando, aceitar e enfrentar a doença, dar maior valor à vida, crer em Deus 
e ter maior esperança de vida. Estes fatores estão entre aqueles que os pacientes deste estudo consideraram significativos para uma vida com qualidade, conforme se observou na Tabela 3.

WHITE (1982) apud GREGG; ROBERTUS; STONE (1989) estudou o nível de satisfação com a vida de 57 adultos diabéticos dependentes de insulina e verificou que não havia diferença significativa com o de pessoas portadoras de doenças não crônicas. Constatou, também, que o nível de satisfação com a vida destas pessoas estava positivamente relacionado com o número de atividades envolvidas (por exemplo: artes, hobbies, viagens, igreja, esporte), com o grau de envolvimento nessas atividades, sucesso no controle da doença, ausência de complicações, e ausência de sinais de depressão ou ansiedade. Tais aspectos também puderam ser observados nas respostas dos entrevistados.

Sete pacientes (15,6\%) consideram-se indiferentes em relação ao diabetes por estarem resignados com a doença e por não serem os únicos com o problema. Outros 07 indivíduos $(15,6 \%)$ referiram estar insatisfeitos, sendo que as causas dessa insatisfação foram: sintomas da doença interferindo na vida pessoal, problemas familiares, e problemas financeiros. Vale ressaltar que somente 01 pessoa $(2,2 \%)$ manifestou estar muito insatisfeita com seu modus vivendi, com a seguinte afirmação: "não posso comer o que desejo e dependo da insulina".

Pudemos constatar, no presente trabalho, que pessoas com diabetes não são necessariamente menos satisfeitas com sua qualidade de vida, fato também observado por LUDMAN; ASPLUND; NORBERG (1990). Estes autores relatam, inclusive, que muitos pacientes perceberam uma influência positiva da doença em suas vidas. Citam, como exemplo, um consumo de alimentos mais nutritivos na família e a adoção de um estilo de vida mais saudável. Relatam, também, que uma proporção significativa de pacientes sentiu que a doença lhes proporcionou um maior desenvolvimento psicológico.

\section{CONCLUSÕES}

Os resultados obtidos demonstraram que, para os pacientes diabéticos, deste estudo, ter qualidade de vida significou prioritariamente ter bem-estar físico $(54,5 \%)$, ter estabilidade sócio-econômica (26,0\%), ter bem-estar psicoemocional e espiritual (16,9\%), enquanto que para outros, o lazer e a auto-estima foram pontos significativos.

Os três aspectos mais afetados pela presença do diabetes, segundo os entrevistados, foram trabalho/estudo/atividades do lar $(38,5 \%)$, capacidade física $(25,6 \%)$ e relacionamento familiar (10,3\%). A limitação física e a necessidade de ausentar-se ou sair mais cedo do trabalho, foram as formas mais referidas de influência sobre o aspecto trabalho/estudo/atividades do lar. Sobre a capacidade física, a interferência ocorreu pela sua diminuição, pelo cansaço físico, 
emagrecimento, fraqueza e desânimo. O relacionamento familiar foi afetado devido ao "nervosismo" que os pacientes relataram pelo fato de terem a doença.

Em relação ao grau de satisfação com a vida, a maioria dos entrevistados considerou-se satisfeita ou muito satisfeita, enquanto que um menor número de pacientes declarou-se indiferente ou insatisfeito com a vida. Apenas um paciente disse estar muito insatisfeito. Foi possivel verificar que o grau de satisfação com a vida relacionou-se, sobretudo, às condições de saúde, à relação familiar harmônica e à resolução de problemas financeiros, familiares e de trabalho.

\section{CONSIDERAÇÕES FINAIS}

Como qualidade de vida entendemos o viver que é bom e compensador em pelo menos quatro áreas: social, afetivo, profissional e a que se refere à saude (LIPP; ROCHA, 1994). O equilíbrio no atendimento das necessidades de cada o desses aspectos pode fazer com que um indivíduo sinta-se bem com a vida.

Os pacientes estudados relacionaram o significado de qualidade de vida mais intensamente com as três áreas a seguir: bem-estar físico, boas condições sócio-econômicas e bem-estar psíquico-emocional-espiritual. É importante salientar que o significado de qualidade de vida é extremamente subjetivo; depende de todo um con texto de vida pessoal, relacionando-se também a fatores como sexo, idade, inserção social, padrão cultural, entre outros. De maneira geral, a maioria dos pacientes declarou estar satisfeita com a vida, mesmo sentindo a influência da doença em áreas como o trabalho/estudo/atividade do lar, capacidade física e relacionamento familiar. Este sentimento de satisfação deve-se, talvez, ao fato de que os indivíduos possuíam compensações que os ajudavam a superar as dificuldades da doença, como por exemplo:

"... eu tenho de tudo, graças a Deus, eu tenho uma família que me apoia em tudo; no caso da doença, eu tenho os médicos a quem eu posso recorrer..."

"... tenho minha família, meus netos que me dão alegria, me dão satisfação."

Ser diabético significa vivenciar uma profunda transformação em seu mundo, aprender a viver com certas limitações e com situações que exigem domínio físico e psíquico de si mesmo. Poucas doenças crônicas requerem do paciente um grau tão elevado de atenção e auto-monitorização quanto o diabetes. A pessoa deve manter sempre um controle do nível glicêmico, uma dieta especial, injeções de insulina e o controle do stress e das atividades físicas. Apesar disso, há sempre a ameaça de descompensação (BAPTISTA, 1992).

Por tudo isso, é fato comum que pessoas diabéticas necessitem também de apoio psicossocial. Tal suporte pode ser oferecido de várias formas e vir da 
família, amigos, outros diabéticos ou profissionais de saúde. O apoio psicossocial tem sido visto como um elemento crítico no sucesso do controle do diabetes. A disponibilidade de apoio da família e amigos está ligada à diminuição da mortalidade e controle da doença (GREGG; ROBERTUS; STONE, 1989).

Como profissionais de saúde, precisamos conhecer estes pacientes em sua totalidade, se o objetivo é de ajudá-los; não devemos restringir nossa atuação apenas aos procedimentos técnicos ou à doença. A crença e o amor à vida e certas potencialidades que a pessoa certamente possui devem estar sendo sempre estimulados, pois da sua atitude positiva é que dependerá, fundamentalmente, a qualidade de vida. É necessário, portanto, que, no contato com estes pacientes, sejamos capazes de ampliar a nossa visão assistencial, integrando a ela a multidimensionalidade do conceito de qualidade de vida.

\section{REFERÊNCIAS BIBLIOGRÁFICAS}

BAPTISTA, M.E.C. Fenomenologia do existir do diabético. Ribeirão Preto, 1992. 99p. Tese (Doutorado) - Escola de Enfermagem de Ribeirão Preto, Universidade de São Paulo.

BEVILAQUA, F. et al . Fisiopatologia Clínica . 4.ed. Rio de Janeiro, Atheneu, 1989.

BRASIL, Ministério da Saúde, Coordenação de doenças cardiovasculares no Brasil- SUS: dados epidemiológicos e assistência médica. Brasil, Ministério da Saúde, 1993.

BRUNNER,L.S.;SUDDARTH,D.S. Tratado de Enfermagem Médico-Cirúrgica. 5.ed. Rio de Janeiro, Interamericana, 1985.

BURCKHARDT, C.S. et al. Quality of life of adults with chronic illness: psychometric study. Res. Nurs. Health, n.12, p.347-54, 1989.

CHACRA,A.R. Diabetes Mellitus. In: PRADO, F.C.;RAMOS, J.A.; VALLE, J.R.do Atualizacão Terapêutica. 15.ed. São Paulo, Artes Médicas, 1991. p. 447-54.

CHATTON. Diagnóstico e tratamento. Såo Paulo, Atheneu, 1983.

CURTIN, M.; LUBKIN, I.M. What is chronicity? In Chronic illness: impact and interventions. 2.ed. Jones ;Bartlett, 1990. Cap.I.

FERRANS, C.E.; POWERS, M.J. Psychometric assessment of the quality of life index. Res. in Nurs. Health, v.15,p.29-38, 1992.

FONSECA, G. T. Modelo para uma classificação de ocupaçōes. Rev. Bras. Est. Pedag. , v. 48,n. 107, p. 274-311, 1967.

GREGG, C.H.; ROBERTUS, J.L.; STONE, J.B. Psychological aspects of chronic illness . Springfield, Charles C. Thomas, 1989. 
GUTIÉRREZ, J.V. La calidad de vida en el cardiopata. Arch. Inst. Cardiol. Méx. v.56. p. 277. $82,1986$.

HELLER, A.Sociologia de la vida cotidiana. 3.ed. Barcelona, Península, 1991.

LIPP,M.; ROCHA, J.C. Stress, hipertensão arterial e qualidade de vida. Campinas, Papirus, 1994.

LUNDMAN,B.;ASPLUND,K.;NORBERG, A. Living with diabetes:perceptions of well being. Res. Nurs.\& Health, n.13, p.255-62, 1990.

MARCONDES, M. et al. Clínica médica: propedêutica e físiopatologia. Rio de Janeiro, Guanabara Koogan, 1989.

THOMPSON, J. M. Quality of life after conventional ileostomy in NSW. WCETJ., p. 8-14, 1990.

TRENTINI, M. et al. Mudanças no estilo de vida enfrentados por pacientes em condições crônicas de saúde. Rev. Gaúcha Enf. v.11, n.1, p.18-28, 1990 (a).

TRENTINI, M. Qualidade de vida dos indivíduos com doenças cardiovasculares, crônicas e diabetes mellitus. Rev. Gaúcha Enf., v. 11, n.2, p. 20-7, 1990 (b). 


\section{ANEXO I \\ Instrumento para coleta de dados}

\section{Dados de Identificação}

Nome:

Siexo: Idade:

Estado Civil: Escolaridade: Ocupação/Profissão:

Situação Empregatícia: Renda Familiar: $\quad N^{\circ}$ de dependentes:

\section{Dados de diagnóstico e tratamento}

Tipo de diabetes:

Tempo de diagnóstico:

Sinais, Sintomas e Complicações:

Regime de tratamento atual:

-Insulina:

-Hipoglicemiante Oral:

-Dieta Alimentar:

-Exercícios Físicos:

\section{Dados sobre a qualidade de vida}

-O que significa qualidade de vida para o (a) senhor (a)?

-Dentre as áreas abaixo relacionadas enumere de 1 a 3 , em ordem de prioridade crescente, as 3 áreas em que o diabetes causou maior influência. Justifique as opções assinaladas.

( ) trabalho/estudo/atividade do lar

( ) auto-imagem

( ) relacionamento familiar

( ) independência /auto-cuidado

( ) recreação e lazer

( ) outros

( ) capacidade física

-De uma forma geral, qual o grau de satisfação com sua a vida atual?

( ) muito satisfeito

( ) Insatisfeito

( ) satisfeito

( ) muito satisfeito

( ) indiferente Por quê?

Entrevistador:

Data: 
SOUZA, T.T. de. et al. Quality of life of the people who suffer from Diabetes Mellitus . Rev.Esc.Enf.USP, v.31, n.1, p. 150-64, apr. 1997.

The purposes of this study were to identify the meaning of the quality of life for the people who suffer from Diabetes Mellitus, to recognize the aspects which affect most their lives due to this disease and the degree of the satisfaction in their lives as well. Participated in this research forty-six (46) diabetic patients, adults of both sexes, who were in a policlinic for treatment. The results showed that the meaning of the quality of life had priority related to the physical well-being $(54,5 \%)$, to the social and economical stability (26,0\%), and to the spiritual and emocional well-being (16,9\%). The most affected aspects due to this disease were: studying and home activities $(38,5 \%)$ physical ability $(25,6 \%)$ and family relationship (10,3\%). Concerning about the degree of satisfaction with their lives, the majority of them $(66,6 \%)$ considered themselves satisfied or very satisfied. It is worth while to point out the importance of considering the multimensionality of the concept of quality of life while attending the diabetic person.

UNITERMS: Quality of life. Diabetes Mellitus. 